\title{
Design and Development of Empire line Denim Kurtis
}

\author{
Vijayalakshmi D \\ \{hod.afd@psgtech.ac.in\} \\ Professor and Head of the Department of Apparel and Fashion Design, PSG College of Technology, \\ Peelamedu, Coimbatore - 641004, Tamil Nadu, India
}

\begin{abstract}
The Denim is an evergreen fashion statement and it is always in the trend at all the seasons. Denim fabrics are used in the manufacture of shirts, jackets, jeans, bags, kids wear. Denim is used in its original form and to add interest various effects like softening, fading, ice washing are rendered during garment washing. Seldom products are seen in the market for woman's knee length tops in denim. This created a spark in designing and developing denim kurtis suitable for Indian market. In this research work, an attempt has been done to use denim fabric with the combination of colorful cotton fabric and develop denim Kurtis suitable for young woman.
\end{abstract}

Keywords: -denim, creativity, dress design, kurtis, young woman.

\section{Introduction}

Denim is sometimes referred to as a "cradle-to-grave" product since it has been worn by people as young as two years old and as old as sixty years old. Denim is one of the oldest materials in the world, but it never goes out of style.Denim garments have an evergreen demand among the consumers globally in all the fashion cycles. Denim is mostly attracted by a group of consumers who loves to be fashionable at the same time focus on durability and low-maintenance costs. The expectation of consumers are increasing and satisfying a consumer is not that easy now-a-days. With the emergence of specialized services, consumers' desire for excellent service and products develops.

Consumers are growing more informed, demanding, and demanding, and their expectations are rising all the time.Young women have become addicted to denim, and the women's clothing industry is expected to rise at a quicker rate from now on, potentially eclipsing the denim market. [1]. The female genders have high involvement and interest towards fashion and they have much more understanding towards clothing than men. Denim began as a workwear garment, but in India, it was discovered to be trendy, pleasant to wear, and appropriate for most situations, as well as providing the requisite style quotient.

With a median age of 26, around 73 percent of India's population is youthful. Even after ten years, the median age will be only 29. Denim wear's target demographic has been 14 to 39 years old, which will account for the majority of the population in the next ten years. In essence, today's consumers, especially the young, are savvier in their fashion choices. They rarely evaluate a product based on a single feature or trait. They frequently evaluate a product using various characteristics. [2].

Designers, product developers, pattern makers, and production engineers are typical members of the garment development team. A design theme can be found anywhere, whether it's in a market, on the beach, or in a skyscraper. As a result, the possibilities for finding 
inspiration are endless, including museums, cities, paintings, sculptures, films, photography, internet and the books. [3].To provide a unique and innovative solutions to design, it is recommended to recruit different creative vocabulary related to theme or concept and organize the same effectively [4]. A collection of unique garments based on the inspiration 'architecture' were designed [5].

\section{Review Of Literature}

\section{A. History of Denim:}

The term "denim" comes from the French term "Serge De Nimes," which refers to a fabric that originated in Nimes, France during the Middle Ages. Denim is the English shot form, according to Webster's dictionary. The blue pigment obtained from indigo dye is used to colour the warp yarn. Indigo was the most important natural dye known to humans until synthetic colours were introduced.[6]

\section{B. Distinctive Feature of Denim:}

Denim's key advantages are its abrasion resistance, the fact that it does not shrink over time after recommended washing, the ability to produce a suitable textured surface, and the fact that it is a fabric that is universally recognised by people of all ages. Denim has a number of flaws, including poor colour fastness and rub resistance; it stretches out, necessitating hot water washing and drying to restore the fit; and it generates thick layers of fabric when sewn.

1) The diagonal surface texture:

The diagonal direction of the twill line might be upward to the right (right-hand twill) or upward to the left (left-hand twill). Furthermore, the floating indigo surface yarns can float over 2, 3, or 4 yarns, resulting in 2-by-1, 3-by-1, or 4-by-1 twill weaves. To add more variety, the width and angle of the diagonal line will be altered. The diagonal lines became more prominent as additional yarns drifted over and the diagonal direction became shallower. The diagonal lines became less noticeable as fewer strands drifted over and the diagonal orientation became steeper.

2) Indigo blue warp yarns and undyed weft yarns:

The diagonal pattern of the surface floating threads is always indigo, and when worn away, the light colour yarns underlying are revealed.

\section{3) Cotton denim:}

According to a poll conducted by cotton incorporate, jeans with a greater cotton content (90 percent cotton or more) have typically commanded a higher price. Consumers perceive the value in paying a little more for a jean that fits better and lasts longer, and 76 percent believe natural fibres produce superior quality clothes. Cotton can be handled roughly during laundering and use because to its strong wet strength. When washing and drying cotton jeans, no particular care is required. When dyed cotton articles are washed in overly hot water, the colour may fade. Warm water should be used to keep the colour from fading. Because chlorine bleach weakens the fibres, it should not be used. [7]. When dyed cotton articles are washed in overly hot water, the colour may fade. Warm water should be used to keep the colour from 
fading. Because chlorine bleach weakens the fibres, it should not be used on a frequent basis. $[8]$.

\section{Types of Denim:}

Denim can be classified based on many aspects like its structure, fibre used, weight and the colour or shade obtained by washing. Among them, colour and weight plays an important role in aesthetic and drape of the garment respectively.

\section{1) Shades of denim fabric:}

The formulas for garment washing jeans are protected by intellectual property laws. To achieve specific looks and fabric hand, several chemicals, hand treatments, and machinery are utilised. These are the three basic washes - raw, medium and light as shown in the Figure 1.

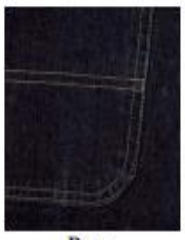

Raw

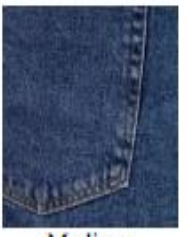

Medium

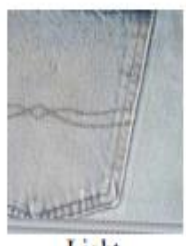

Light

Fig.1 Three basic washes of denim fabric

The majority of the dye is preserved, and softeners may be added to the resin finish to soften it. Chemicals for bleaching and softening are added in Medium wash; this takes a longer washing period until enough indigo dye seeps out to achieve the medium blue colour. Chemicals for bleaching and softening are introduced in light; this takes the most washing time and dye loss to get the light blue colour..

\section{2) Weight of denim fabric}

Denim is available in a range of weights. It comes in a variety of weights, from classic 12 oz. and $14 \mathrm{oz}$. per yard to a soft, drapey $6 \mathrm{oz}$. version. When it comes to jeans, jackets, overalls, and work items, heavy denim (12 to 14 ounce) works best. It's heavy and stiff, so it's perfect for patterns with straight lines and little to no gathering or easing. Medium-weight (10 to 12 ounce) denim works nicely for pants, jackets, skirts, and accessories like purses. Lighter denim is more forgiving of softer style and curved seams that require relaxing and/or gathering. Lightweight denim works well with skirts, shirts, and dresses (6 to 8 ounce). This denim is the easiest to work with and stitch.

\section{Sewing of Denim:}

Denim should be treated like any other tightly woven fabric. Larger sewing machine needles (size 16 or 100) and a longer stitch length are utilised for heavy-woven denims (10 stitches per inch). A lower needle size, 11 or 14 (70 or 80$)$, and 12 stitches per inch are utilised for lighter weight denims. The fabric feeds uniformly when the thread tension and pressurefoot pressure are reduced.[9] 

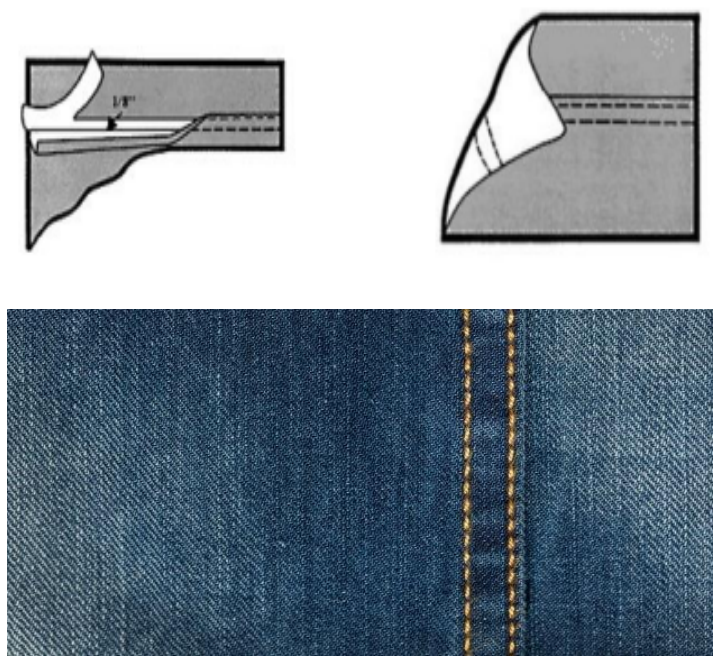

Fig 2. Flat felled seam

In denim apparel, the flat-felled seam as shown in Figure 2 is the most common used seam. It's tough and it gives a neat and polished look. Most seams can be flat-felled, but only if the armhole seam has a shallow sleeve cap, such as a shirt sleeve, can it be flat-felled.
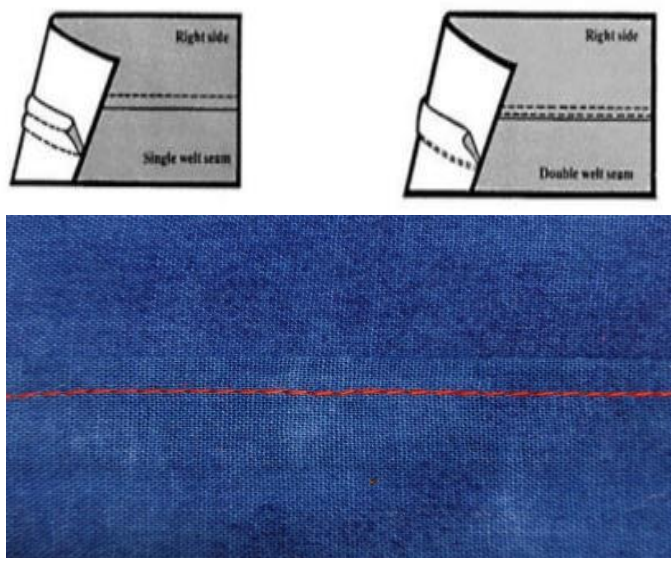

Fig 3. Single and double welt seam

Welt seams illustrated in the Figure 3 works well for heavyweight denim and have nearly the same appearance as the flat-felled seam. Fringed seams can be utilised on the sides and yokes of pants. An inside-out seam is a form of ornamental seam. Depending on the style, matching or contrasting thread is utilised. Denim topstitching can add a lot of stylish appeal, but only if done correctly. Topstitching with a thick contrasting thread, such as buttonhole twist, can be done in single or several rows. Regular weight thread should be used on the bobbin, and the top tension should be decreased to accommodate the heavier thread. The threads will float on top of the fabric as the stitch length is increased. [10] 


\section{F.Denim Garment Washing and Finishing:}

Denim fabrics have been washed using different garment washing processes to generate a wide range of fashionable fashion denim clothing. On denim items dyed with traditional indigo dye, which is chosen for its beautiful blue colour and low wash fastness, a variety of washing effects can be achieved. The exhaustion approach is the optimum way for finishing at the garment stage. Enzyme finish is used to soften denim clothes. The microencapsulation process improves the functional finish's durability.

\section{G. Design and development of denim kurtis:}

Many people are involved in the process of apparel development like textile, fashion designers, pattern makers, manufacturers. The designer kickstarts her or his work with an inspiration for the conceptfrom anywhere. Therefore the sources of inspiration are unlimited such as street side vendors, museums, cities, paintings, sculptures, films, photographs, books and internet. The inspiration has been 'civilised environment'in developing a collection [11]. A collections of unique garments based on the inspiration 'architecture' were designed by Halime Paksoy \& SemaYalçon [12]. Denim tops are making a fashion statement in and of itself, and the Denim Club India has predicted that they would be the trend for the coming season. The trend setters will be full-sleeved shirts for office workers and stylized tops for college students, so the denim top will be seen and included in many wardrobes [13]. A mood board is the initial step in putting a designer's ideas and images together into a concept. This allows them to channel their creative energy into a unified and targeted design outcome. Images and colour concepts are arranged on a huge board to create mood boards. A designer can also use the process of creating a mood board to gather visual information from a range of sources and organise their inspirations and ideas into a composed visual presentation. Mood boards can be made on paper or on a computer. [14]

Close analysis of the source of inspiration's surface detail will elicit intriguing ideas about textures and colours, which will affect the designers' fabric selection. Instead of relying on commercially available fabrics, designers can create their own textiles using techniques such as embroidery, dyeing, knitting, and printing. These one-of-a-kind textiles can subsequently be used to create really one-of-a-kind garment designs [14]. In the business world, colour boards are used to clarify specific colours and combinations within a product category or retail theme.A palette is a limited palette of colours that a designer utilises in a collection to guarantee that all of the colour aspects are balanced. It's always a good idea to have at least 10 - 12 colours in your palette for a season. $[15,16]$.

Any designer's portfolio should include fashion illustration boards. Croquie are used to drape the clothing in the illustration. Fashion sketching, which is usually the act of drawing the dressed figure on a stylised human shape, requires a thorough understanding of the human body (croquie). Depending on the desired impact, fashion figures can be cropped and framed on drawing boards. In order to communicate the type of fabric to the buyer, texture and colour rendering are also highly significant in drawing. Denim fabric, for example, is depicted with diagonal strokes to emphasise the twill weave. [17]. Technical drawings are created to illustrate an understanding of individual clothes, such as stitching and seams, fasteners, pocket placement, and so on. Technical specifications should always be drawn in a clear and linear style: they must be accurate and detailed enough for a factory manager or garment 
technologist to understand them, and they must include enough information for a sample machinist to assemble the garment without further instruction.[15,16]

\section{Methodology}

The product development set in motion with an inspiration and thereafter development of mood board, colour and fabric board, illustration board, pattern making, garment construction and finally presentation of the denim kurtis on mannequins.

\section{A. Source of Inspiration and Mood Board Creation}

The inspiration for the development of denim kurtis for young woman was obtained from 'blossom'. Flower is one of the most admired aspects in the world. The bright coloured flowers with the blossomed petals have been a source of inspiration for the current study for the line, colour, shape, form and texture of a design. Spring season

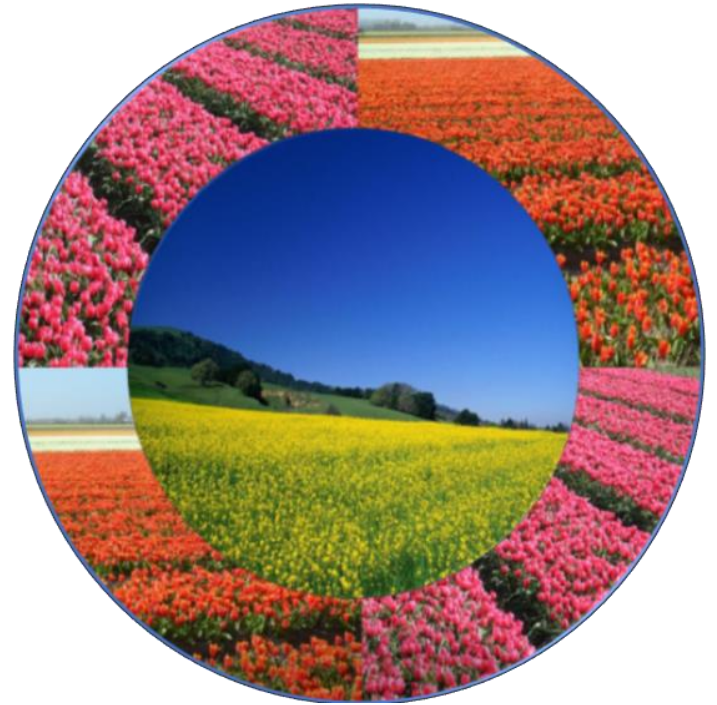

IFig.4 Mood board developed based on the concept "Blossoms"

gives life and freshness to the world as it arrives after the cold winter in which it seemed that everything was dead. The world is filled with colour and the scent of blossomed flowers. In order to enhance the beauty and give life to a whole new clothing dimension of denim, the category chosen was young women's top using lightweight denim. Hence the concept of blossom has been used to give life and elegance to the new era of clothing using denim. A mood board was developed using the pictures of sky, blossomed flowers from the fields. The developed mood board is given in the Figure 4.

\section{B. Colour and Fabric Board Development}

The colours inspired from the mood board for the design development were shades of green, yellow, blue and purple.Each colour has got its own uniqueness and properties. Green was obtained from the leaves and it indicates refreshing, natural and newness. 


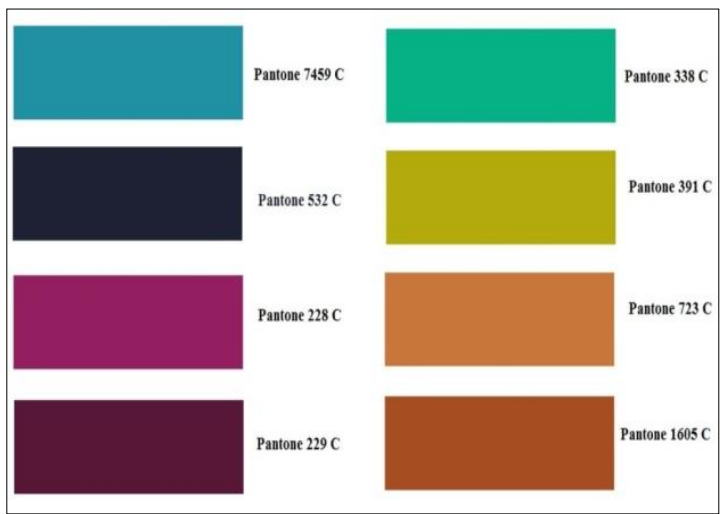

Fig.5 Colourboard developed

Yellow was obtained from the sun and it indicates liveliness, cheerfulness and brightness. Blue is obtained from the sky and it indicates soothing, calmness and cool. Purple is obtained from the flowers and it indicates the richness and royalty.

From the Figure 1, the streaks of flowers in the form it is cultivated were highly inspired and line was the dominant element obtained as inspiration from the mood board. The design of the fabric inspired from the mood board was self-striped $100 \%$ cotton fabrics that could coordinate with light weight denim. The fabrics finally selected for the construction of denim kurtis were $100 \%$ cotton light weight denim, $100 \%$ cotton fabric and it is depicted in the figure 6.

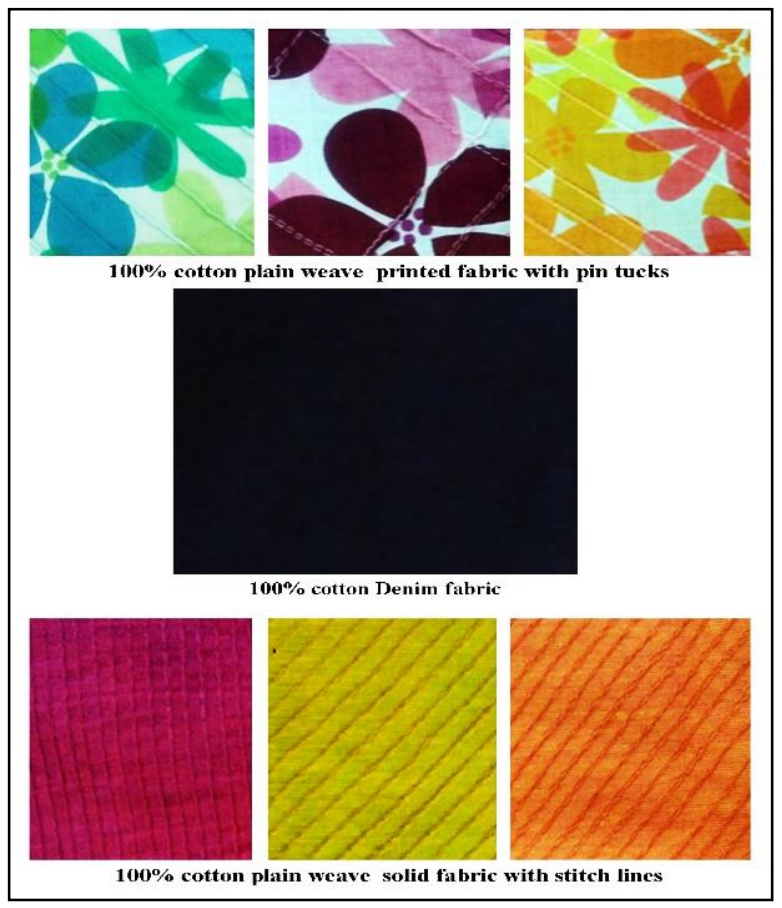

Fig.6Fabric board developed 


\section{Illustration Board Development}

Since the design collections were focused on denim kurtis for young woman, medium shots (the torso of female croquie) were done for the illustration boards. The silhouette of the garment collections was selected as empire line. Five designs were developed based on the inspiration. The designs were given style code as denim empire line kurtis (DELT 01, 02, 03, $04 \&$ 05). The developed designs are given in the Figure 7. The illustrations were rendered on $10 \frac{1}{2}$ head theory based female croquie. Blue diagonal lines were used to render the denim fabric.

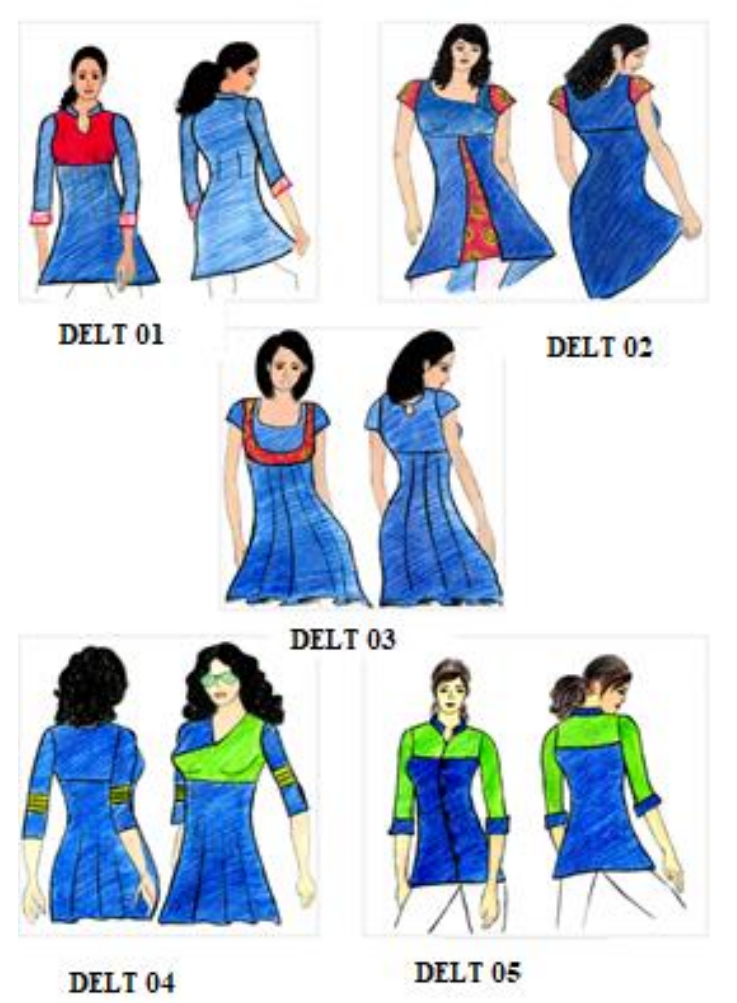

Fig.7Illustration baord developed

The design with style code DELT 01 has a simple yoke and wide cuff with contrast fabric. The neck is finished with a Chinese collar. DELT 02 is asymmetrically balanced garment and it has an inverted box pleat at the lower bodice. DELT 03 has a contrast yoke in the front and flared out at the bottom. DELT 04 has asymmetrical overlapping yoke using denim coupled with contrast coloured fabric. The middle part of the sleeve is also done with a contrast coloured fabric to create a harmony. The last design is DELT 05 with a simple yoke continuous placket and three fourth sleeves with contrast cuff. The neck is finished with a Chinese collar.

D. Pattern making and Garment construction 
Pattern making was done using drafting technique. Each design of denim kurtis were carefully analysed for its style features. The technical drawings shown in the figure 8were prepared for the same. With the guidance of the technical flats, the patterns were developed. The pattern details like name of the pattern block, cut number and grain line were incorporated inside the pattern blocks. The operation sequence for the construction of denim kurtis were meticulously planned based on the quality specification and ease of material handling.
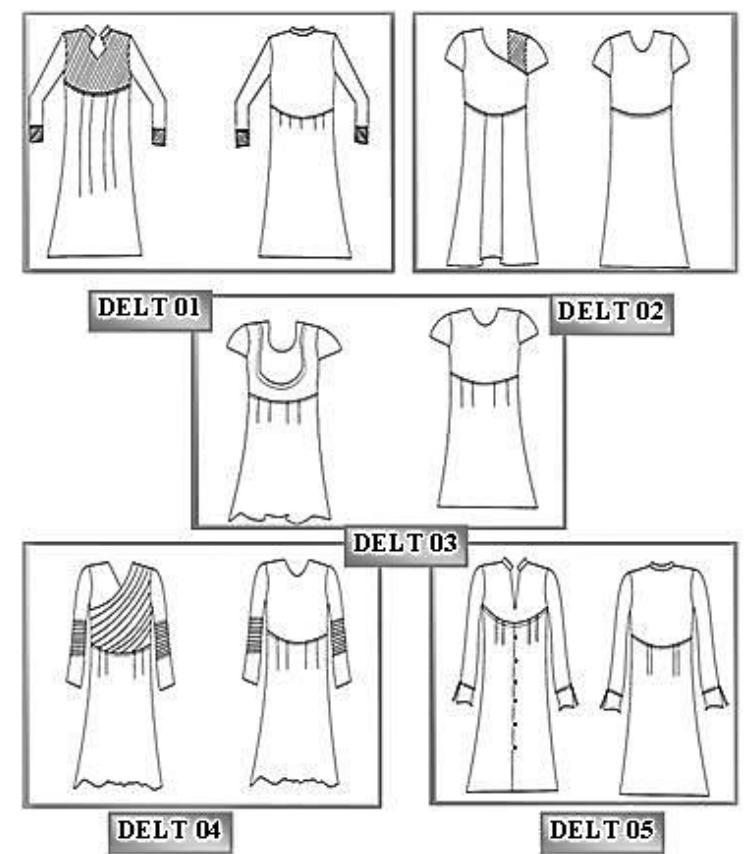

Fig.8 Technical Drawing

All the patterns were cut in straight grain other than the collar and cuff blocks. The front and back centre panel was cut one each and the side panels were cut four. The sleeve pattern was cut two and the ruffles at front placket, cuff and collar was cut in contrast coloured fabric. Single needle lock stitch machine and over lock sewing machines manufactured by Juki was used for construction.

The patterns and the operation sequence of the denim empire line kurti DELT 01 is given in the Figure 9and 10 respectively.

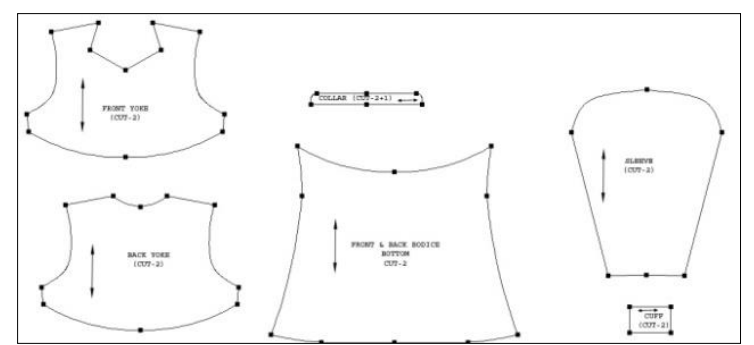


Fig.9. Pattern blocks of denim empire line kurti DELT 01

All the patterns were cut in straight grain other than the collar and cuff blocks. The front and back bodice were cut one each. Yokes were cut four pieces, two for front and two for back. The sleeve pattern was cut two, cuff and collar was cut in contrast coloured fabric.

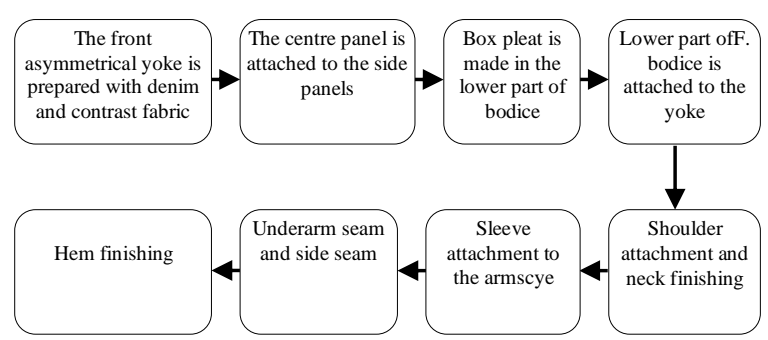

Fig.10Flow chart of operation sequence for denim empire line kurti DELT 01

The patterns and the operation sequence of the denim empire line kurti DELT 02 is given in the Figure 11 and 12 respectively.

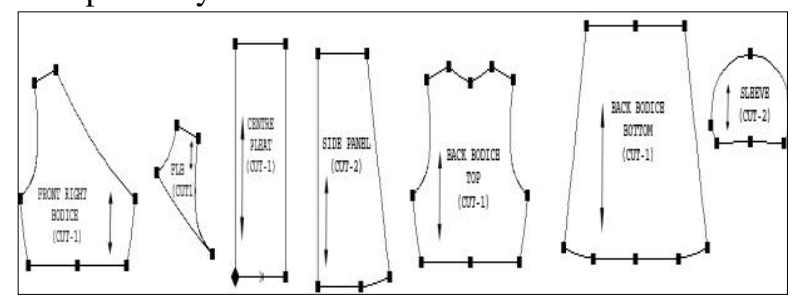

Fig.11 Pattern blocks of denim empire line kurti DELT 02

All the patterns are cut in straight grain. The front yoke is asymmetrical and it was cut two, one in denim and other one in contrast fabric. Thin contrast detailing was given at the bottom of the yoke. The sleeve was cut two and has a contrast hem. Back yoke and its bodice were cut in denim fabric.

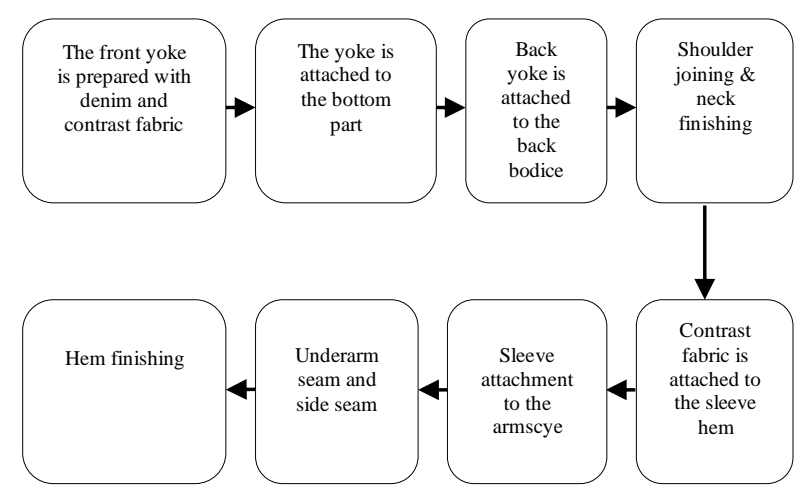

Fig. 12 Flow chart of operation sequence for denim empire line kurti DELT 02 
The patterns and the operation sequence of the denim empire line kurtis DELT 03 is given in the Figure 13 and 14 respectively.

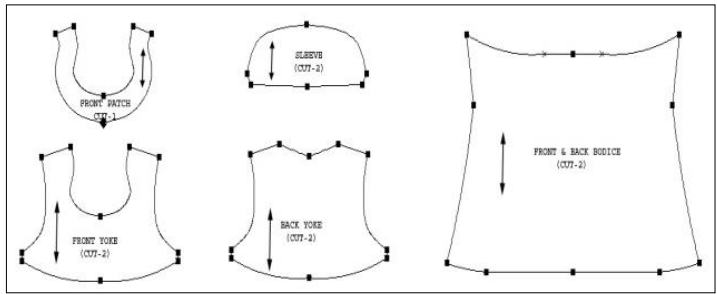

Fig.13 Pattern blocks of denim empire line kurti DELT 03

All the patterns were cut in straight grain. Two pieces of front yoke was cut in contrast fabric. The patch in the front yoke was cut in denim fabric. The sleeve was cut two and has a contrast hem. Back yoke was cut two in contrast fabric and its bodice was cut in denim fabric.

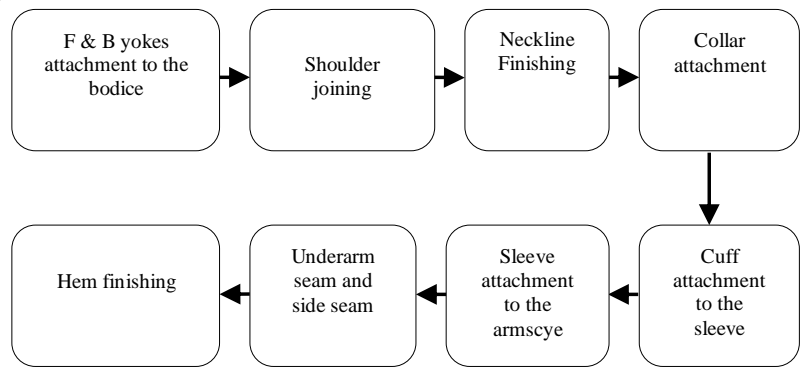

Fig.14Flow chart of operation sequence for denim empire line kurtis DELT 03

The patterns and the operation sequence of the denim empire line kurti DELT 04 is given in the figure 15 and 16 respectively.

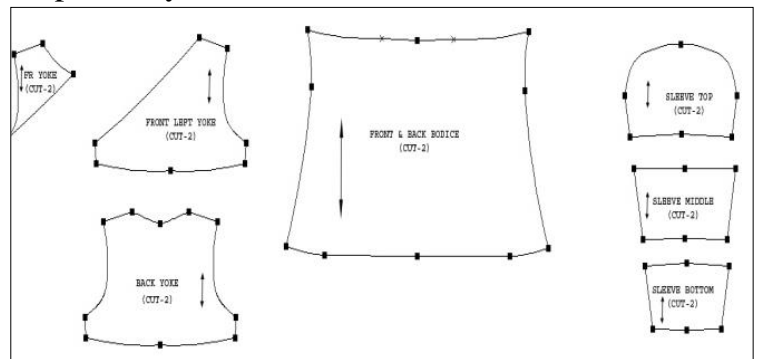

Fig 15 Pattern blocks of denim empire line kurti DELT 04

All the patterns were cut in straight grain. Front yoke was divided into two parts, the wider one was cut in contrast fabric and the smaller one was cut in denim fabric. The sleeve is divided into three parts, top and bottom portion in denim and middle portion in contrast fabric.

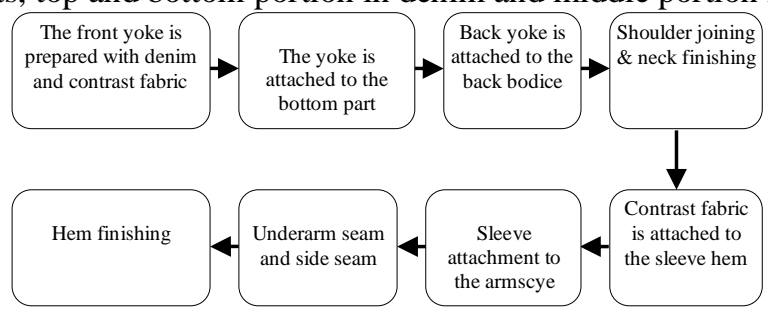


Fig. 16 Flow chart of operation sequence for denim empire line kurti DELT 04

The patterns and the operation sequence of the denim empire line kurti DELT 05 is given in the Figure 17 and 18 respectively.

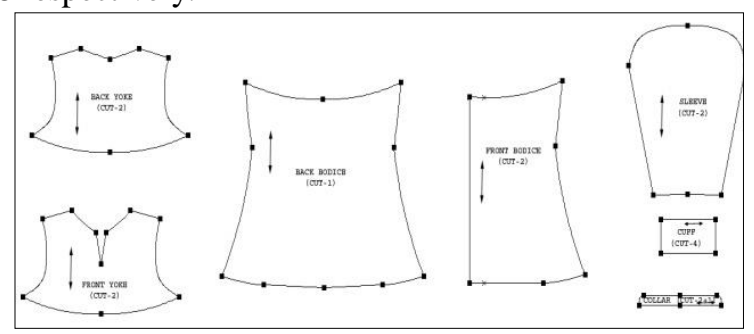

Fig. 17Flow chart of operation sequence for denim empire line kurti DELT 05

All the patterns were cut in straight grain other than cuff and collar. Front and back yokes were cut in two numbers each in contrast fabric. The front bodice was cut two to place the buttons in the front and the back bodice is cut one. The sleeve and the collar were cut in denim fabric. The cuff was cut in the contrast fabric.

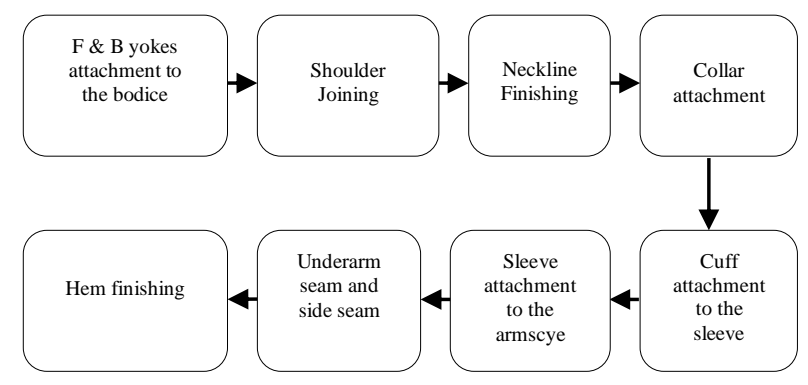

Fig.18 Flow chart of operation sequence for denim empire line kurti DELT 05

\section{E. Presentation of the Denim kurtis:}

The developed denim kurtis were draped on the mannequin for the final presentation. The front and back pose of the mannequin with the developed denim garments were shot using the camera.

Figure 19 portrays the developed princess line denim kurtis DPLT 01, 02, 03, 04 and 05 presented in the mannequins.Figure 19 portrays the developed empire line denim kurtis DELT 01, 02, 03, 04 and 05 presented in the mannequins. 

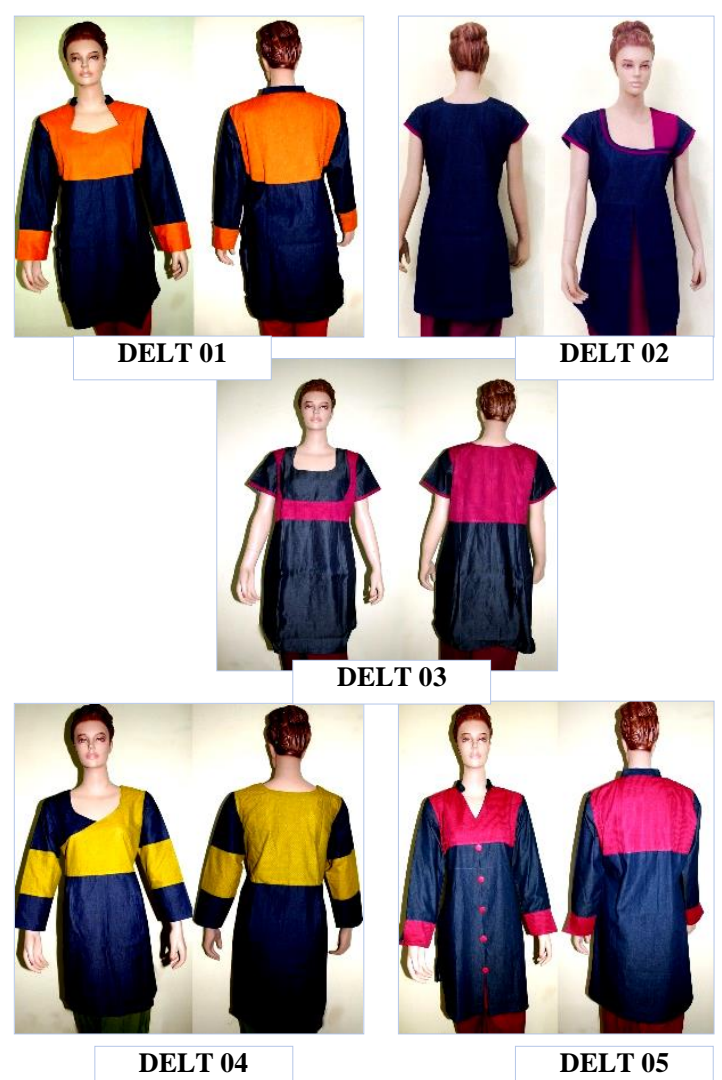

Fig. 19 Final presentation of denim empire line kurtis (DELT) - front \& back view

The developed denim kurtis were draped on the mannequin for the final presentation. The front and back pose of the mannequin with the developed denim garments were shot using the camera.Figure 3 portrays the developed empire line denim kurtis DELT 01, 02, 03, 04 and 05 presented in the mannequins.

F. Feedback from the experts:

Qualitative research method was used to obtain the feedback of the developed denim kurtis. Twenty experts were selected opine about the developed denim kurtis. A questionnaire was prepared to rank the colour combinations, fabric combinations, design details used in the denim kurtis and overall aesthetic of the denim top and their suitability to the South Indian market. The ranking were obtained using Likert scale $(1-5)$ where, 5 was ranked as very good; 4 as good; 3 as average; 2 as poor and 1 as very poor. The filled questionnaire was consolidated and the percentage of the experts' opinion based on the rank given to each criterion in the questionnaire. 


\section{Results And Discussion}

The results obtained are consolidated and are tabulated in Table 1.In empire line denim kurtis, the colour combination used was ranked as very good by $65 \%$, good by $20 \%$, average by $15 \%$ of the experts. The combination of denim fabric with coloured cotton fabric was ranked as very good by $70 \%$, good by $15 \%$ of the experts. The design details of the developed denim kurtis like length, silhouette, yoke, sleeve and collar were ranked as very good in the range of $55 \%$ to $70 \%$ by the experts. The overall aesthetic of the garment developed were ranked by the experts as very good by $70 \%$, good by $20 \%$ and average by $10 \%$. The suitability of the developed denim market to the southern market especially Coimbatore isranked by the expert as very good by $75 \%$, good by $15 \%$ and average by $10 \%$.

TABLE 1

PERCENTAGE OF EXPERTS’ OPINION ON DENIM EMPIRE LINE STYLE

\begin{tabular}{|c|c|c|c|c|c|c|}
\hline \multirow[t]{2}{*}{ S.No. } & \multirow[t]{2}{*}{ Particulars } & \multicolumn{5}{|c|}{$\begin{array}{l}\text { Rating by Experts } \\
\text { (percentage) }\end{array}$} \\
\hline & & 5 & 4 & 3 & 2 & 1 \\
\hline & $\begin{array}{l}\text { The colour } \\
\text { combination used in } \\
\text { the developed denim } \\
\text { kurtis }\end{array}$ & 65 & 20 & 15 & 0 & 0 \\
\hline & $\begin{array}{l}\text { The combination of } \\
\text { the fabric used in the } \\
\text { developed denim } \\
\text { kurtis }\end{array}$ & 70 & 15 & 10 & 5 & 0 \\
\hline & \begin{tabular}{l|l} 
The & Length
\end{tabular} & 55 & 40 & 5 & 0 & 0 \\
\hline & Silhouette & 60 & 40 & 0 & 0 & 0 \\
\hline & details & 50 & 35 & 10 & 5 & 0 \\
\hline & used in & 70 & 30 & 0 & 0 & 0 \\
\hline & $\begin{array}{l}\text { the } \\
\text { developed } \\
\text { denim } \\
\text { kurtis }\end{array}$ & 60 & 35 & 5 & 0 & 0 \\
\hline & $\begin{array}{l}\text { Overall aesthetic of } \\
\text { the developed denim } \\
\text { kurtis }\end{array}$ & 70 & 20 & 10 & 0 & 0 \\
\hline & $\begin{array}{l}\text { Suitability of the } \\
\text { developed denim } \\
\text { kurtis to the southern } \\
\text { market especially } \\
\text { Coimbatore }\end{array}$ & 75 & 15 & 10 & 0 & 0 \\
\hline
\end{tabular}




\section{Summary And Conclusion}

An attempt has been made to design and develop a novel product using denim fabric for young woman. A mood board was developed based on the "Blossom" concept. A collection of Denimempire line kurtis were designed. Patterns were made using drafting technique. The patterns were cut in the specified fabrics and developed into garments. The denim kurtis were evaluated by a panel of 20 experts. A questionnaire was given to the experts, the feedback obtained were consolidated. The results have proved that the developed denim kurtis are meeting its design standards and have high potential in south Indian market.

\section{References}

[1] Amit Gugnani, (2011), Denimwear market in India reaching to tier-II and III cities. Available from <http://www.technopak.com/Files/ Denimwear_market_in_India.pdf > [1 July 2010].

[2] Osmud Rahman (2011), 'Understanding consumers' perceptions and behaviours: Implication for denim jeans design', Journal of textile and Apparel Technology and Management, vol. 7, no.1, pp. $1-16$.

[3] Elaine Stone (2004), Dynamics of Fashion, Fairchild Publications, New York.

[4] Suhaila Hassan Al-Montser Al-Yamani \&HananAbdulhalim Baw Bukhari (2011), 'Civilized environment as a source of Inspiration in Fashion Design', Journal of textile and apparel, technology and management, vol.7, no.1, pp.1-14.

[5] HalimePaksoy\&SemaYalçın, (2005), 'Architectural Inspirations in Fashion Design', Proceedings of the 3rd international symposium of interactive media design, pp.1-9.

[6] (http://www.denverfabrics.com/content/denim/denim.aspx. www.denverfabrics.com [June 2010]

[7] Cotton incorporate supply Insights, 2011, "Denim jeans - the state of us market, Cotton Incorporated

[8] Kadolph, SJ 2010. Textiles, Upper Saddle River, Prentice Hall, New Jersey

[9] https://www.singer.com/sewing-resources/choosing-the-right-machine-needles

[10] Rose Marie Tondl, Extension Clothing Specialist, "Sewing with Denim ", DigitalCommons at University of Nebraska - Lincoln, 1989.

[11] Suhaila Hassan Al-Montser Al-Yamani \&HananAbdulhalim Baw Bukhari, "Civilized environment as a source of inspiration in Fashion design", Journal of Textile and Apparel Technology Management, vol.7, no.1, pp.1-14.

[12] Halime Paksoy \&SemaYalcon, "Architecutral Inspirations in Fashion Design", Proceedings of the 3rd International Symposium of Interactive Media Design, pp.1-9, 2005.

[13] Denim Club India (DCI), $\quad$ News 2010. http://www.denimclubindia.org/nltr/dciNewsletter_Jan2010.asp.,5 January 2011.

[14] Kathryn McKelvey\& Janine Munslow, "Fashion design process, innovation and Practise", Balckwell Publishing, Oxford, UK 2010.

[15] Caroline Tatham\& Julian Seaman Fashion Designing and Drawing Course, Thames and Hudson Publishers, UK, 2003.

[16] John Hopkins, "Fashion Drawing", AVA Book Production Pte. Ltd., Singapore. 2010.

[17] RituBarghav, Fashion Illustration and Rendering, Jain Publishers (P) Ltd, New Delhi, India. 